\title{
Hearing Impairment in Pediatric Patients with Down's Syndrome; A Systematic Review of Literature
}

\author{
Rawya Ibrahim Abuelhassan Abdalla ${ }^{1} \quad$ Moustafa Abdualmonuim Ahmed ${ }^{2}$ \\ 1. Emirates Specialty Hospital \\ 2.Ministry of health, Almadam Health Centre
}

\begin{abstract}
Even though hearing loss (HL) is common in children with Down syndrome (DS), there has been little research investigating the effects of hearing loss. The purpose of this review is identification of hearing impairment and common type of hearing loss in Down's syndrome pediatric population. A systematic review was performed during period of 10 months. A thorough search was conducted by using key words Trisomy 21, Down's syndrome, Sensorineural Hearing loss, Pediatrics, Audiology, Speech and Hearing sciences. 22 relevant articles were reviewed and it showed the evidence that conductive hearing loss is more prevalent in DS population as compared SNHL. However, longitudinal studies on large populations are required.
\end{abstract}

Keywords: Down's syndrome, Hearing impairment, sensorineural hearing loss, Conductive hearing loss, Trisomy 21.

DOI: $10.7176 / \mathrm{JHMN} / 96-04$

Publication date: December $31^{\text {st }} 2021$

\section{INTRODUCTION}

Down syndrome (DS) is the most common chromosomal malformation in newborns. In Europe, DS accounts for $8 \%$ of all registered cases of congenital anomalies. Throughout the world, the overall prevalence of DS is 10 per 10,000 live births, although in recent years this figure has been increasing. To a large extent, the prevalence of DS depends on several socio-cultural variables. In countries where abortion is illegal such as Ireland and the United Arab Emirates, its prevalence is higher. Conversely, in France, DS prevalence is low, and this is probably due to a high percentage of DS pregnancy terminations ${ }^{1-3}$. In The Netherlands, the most recent measure of DS prevalence was 16 per 10,000 live births ${ }^{4}$. In the United Kingdom, the prevalence of pregnancies affected by DS has increased significantly, but there has been no overall change in the live birth prevalence of DS. Increasing maternal age and improved survival rates for infants with Down syndrome have outweighed the effects of prenatal diagnosis followed by the termination of pregnancy and a declining general birth rate ${ }^{1,3,5-7}$.

DS is characterized by several dysmorphic features and delayed psychomotor development. Children with DS also have an increased risk of concomitant congenital defects and organic disorders such as congenital heart and gastrointestinal defects, celiac disease and hypothyroidism ${ }^{2}$. The median age at death of individuals with DS has risen significantly in the US, from 25 years in 1983 to 49 years in 1997. Congenital heart defects (CHD) and respiratory infections are the most frequently reported medical disorders on death certificates for individuals with DS ${ }^{8} .38$ Standardized mortality odds ratios (SMORs) in DS were low for malignancies except for leukemia and testicular cancer, which were seen more often in individuals with DS ${ }^{2,9}$. Recent decades have seen a substantial increase in the life expectancy of children with DS. In The Netherlands, the infant mortality rate in children with DS dropped from $7.07 \%$ in 1992 to $4 \%$ in 2003 (this is in contrast with the $0.48 \%$ infant mortality of the reference population in The Netherlands in 2003) The fall in DS mortality was mainly related to the successful early surgical treatment of CHD and to the improved treatment of congenital anomalies of the gastrointestinal tract ${ }^{4}$. The life expectancy of children with DS is primarily dependent on the risk of mortality in the first year of life. While modern medical care has reduced the mortality rate to more acceptable values, both morbidity and mortality could be further reduced. In this respect, respiratory infections and neonatal problems are the most important issues to be solved. Since children with DS now have an improved life expectancy, the total population of individuals with DS is expected to grow substantially. Preventive health care programmes for these children will contribute to the improvement of their overall outcome and quality of life; therefore, it is very important to keep the medical guidelines updated. ${ }^{2,10}$

Hearing impairment and otologic problems are prevalent in children with DS, and these problems correlate substantially with developmental problems. Midface hypoplasia is common in children with DS. Mid-face problems, together with hypotonia and macroglossia (children with DS have a relatively large tongue compared to the oral cavity), are responsible for chronic middle ear disease and chronic rhinorrhoea. Mild hearing loss can influence educational, language and emotional developments, and as a result, it can affect a child's articulation skills. Regular assessment of the hearing function is very important. An active search for and treatment of chronic ear disease in children with DS, started soon after birth, may improve hearing. ${ }^{11}$

The rationale of this review is to summarize the literature available to determine the type, causes and incidence of hearing loss in Down's syndrome pediatric population for the purposes of informing clinical 
services and guiding future research.

\section{METHODOLOGY:}

A literature review was planned and performed using methods specified in the Preferred Reporting Items for Systematic Reviews and Meta-Analyses (PRISMA) guidelines. Initial search terms were compiled and iteratively refined by content experts in the fields of Otology, Neurotology, \& Cranial Base Surgery; Speech, Language, and Hearing Sciences; Library Science, Pediatrics and Geriatrics. Both controlled vocabulary terms (e.g. $\mathrm{MeSH}$ ) and key words were used to search the following databases for articles related to Down's syndrome, hearing. DS in Pediatrics, Trisomy 21 and sensorineural hearing loss: PubMed/ MEDLINE (1980-2021), Wiley/Cochrane Library (1970 - 2021), Thomson-Reuters/Web of Science (1970-2021) EBSCO/PsycINFO (1980's-present), and EBSCO/CINAHL (1981-2021) Inclusion criteria were

- Population: the study had to include Down's syndrome pediatric population

- Studies involving adult populations were excluded

\section{RESULTS}

After reviewing multiple articles 22 studies met inclusion criteria.

The pinna in the Down's syndrome child is 2 SD smaller than the general population ${ }^{12}$. This has the effect of decreasing sound localization and sound concentration. Not only is the pinna small, but the external auditory canal is often stenosed (in up to $50 \%$ of children with DS) ${ }^{13}$. This can make examination of the ear difficult for the General Practitioner leading to missed effusions, and also predisposes the child to cerumen impaction, which can lead to a conductive hearing loss (CHL). As such, Shott et al recommended that children with stenotic canals be assessed by an Otolaryngologist every three months until the age of 2-3 when their canals have grown to ensure effusions are not missed. In addition, regular cerumen debridement of ears in patients where cerumen impaction poses a problem is recommended to improve visualization of the tympanic membrane. ${ }^{14}$

Down syndrome children have an increased rate of chronic ear disease ${ }^{13,14}$ the causes are likely multifactorial. The incidence of chronic ear disease in the normal pediatric population has not been well defined. Study conducted by Austeng et al concluded that both conductive and sensorineural hearing loss is still common in children with Down syndrome children at the age of eight and as much as two thirds of the children may have a bilateral impairment. These participants require a continuous audiological follow-up throughout childhood. ${ }^{15}$ Casselbrant et al. conducted monthly examinations using otoscopy and tympanometry in children between the ages of two and six and found that $53 \%$ of the children had at least one middle ear effusion in the first year; this had increased to $61 \%$ in the second year ${ }^{16}$.A recent study showed the prevalence of OME at age one is up to $93 \%$ while at age 5 , the prevalence is up to $68 \%$ in the DS population ${ }^{17}$. In DS patients, up to $80 \%$ will have conductive hearing loss as a result of OME 18.

Study conducted by Nightengale et al showed permanent hearing loss in $24.9 \%$ of the children, among whom bilateral (75.4\%) and conductive (33.3\%) hearing losses occurred most often. Of children with DS, $22 \%-$ $30 \%$ experienced a transient hearing loss, with a high incidence of middle ear pathologies from infancy until early adulthood. ${ }^{19}$ Park et al. showed a $37.9 \%$ rate of CHL due to serious otitis media in a study analyzing 323 DS patients ${ }^{20}$ A prospective observational study by Schwartz et al. concluded that the Eustachian tube has been found to be smaller on average in DS children ${ }^{12}$ As well, the generalized hypotonia in DS patients impacts the function of the tensor veli palatini muscle, which is felt to lead to poor Eustachian tube opening and subsequent poor aeration of the middle ear space ${ }^{12,14}$ Park et al evaluated auditory brain-stem responses of 47 unselected patients 2 months to $31 / 2$ years of age. Thirty-four percent had normal hearing, $28 \%$ had unilateral loss, and $38 \%$ had bilateral loss. Type of loss was conductive in 19 ears, mixed in 14, and sensorineural in 16. Degree of loss was mild in 33 ears, moderate in 13, and severe to profound in 3. Language quotients and degree of hearing loss were compared and an association was not found. Because of the high prevalence of hearing loss in children with Down syndrome, we recommend evaluation of auditory brain-stem responses in the first 6 months of life. ${ }^{20}$ Sensorineural hearing loss (SNHL) can also be an issue with a child who has Down Syndrome. The incidence is not clearly defined; the incidence ranges from 4 to $55 \%$ in DS children ${ }^{21-23}$. The prevalence and causes of sensorineural hearing loss (SNHL) in children with Down syndrome (DS) are poorly delineated. A crosssectional study was performed among all children with DS followed by Schrijver et al among 291 patients in follow-up, 138 patients $(47.4 \%)$ presented with hearing loss. In the majority this was caused by middle ear effusion and only 13 patients $(4.5 \%)$ had sensorineural hearing loss. Hearing loss severity was graded as mild in $38.5 \%$, moderate in $30.8 \%$ and profound in $30.8 \%$ of the patients. Sensorineural hearing loss is uncommon in children with DS with a prevalence of $4.5 \%$. Etiological work-up may allow identifying a specific underlying cause. Cochlear nerve deficiency was found in 4 children with DS and single sided deafness. ${ }^{24}$

Roizen at el evaluated 73 children evaluate at the clinic, 47 underwent ABR evaluation at Siegel Institute and are included in this study. Of the 47 children with DS, 16 (34\%) had normal hearing bilaterally, $13(28 \%)$ had unilateral loss, and 18 (38\%) had bilateral loss. Regarding the 49 ears with hearing loss (52\%), type of loss 
was conductive in 19 ears; mixed in 14 ears, and sensorineural in 16 ears. Degree of loss was mild in 33 ears, moderate in 13 ears and severe to profound in 3 ears. Tympanometric testing produced reliable results in 28 of the 47 children with DS. Tympanometric results confirmed hearing loss as conductive in 9 patients, mixed in 8 , and sensorineural in 5. ${ }^{25}$ Shott et al. showed that only $2 \%$ of DS patients had persistent hearing loss after treatment of hearing loss secondary to chronic otitis media with pressure equalization (PE) tubes ${ }^{14}$. However, conflicting studies have also showed that DS patients did not experience the same benefit from PE tubes as a control group. Similar to the general population, observation is the recommended first line treatment for the first 3 months after diagnosis. PE tubes are indicated in cases of severe hearing loss, if there was a risk of pathological changes of the eardrum, or if there is failure of the effusion to clear after 3 months. Often, insertion of PE tubes is delayed until the ear canal is large enough to allow for insertion ${ }^{26}$. Paulson et al. recently showed that out of their 102 patients with DS, 63.7\% required two or more sets of tubes during their follow up period and that long term complications such as perforation, atelectasis and cholesteatoma were much more common in this population ${ }^{27}$. Since hearing loss in the Down syndrome population is very common, early screening and close monitoring can ensure optimal outcomes. The guidelines from the American Academy of Pediatrics suggests audiological testing at birth (ABR or OAE), testing every 6 months until the age of three and then annual testing thereafter ${ }^{28}$

\section{DISCUSSION}

Children with Down's syndrome are at a greater risk of developing a hearing impairment. Raut et al reported the incidence of hearing loss in the first year of life at $34.1 \%$. Frequent hearing assessments should be undertaken to identify and treat this often reversible hearing disability ${ }^{29}$. International guidelines recommend testing with otoacoustic emissions (OAE), followed by automated auditory brainstem response (AABR) within six weeks 30 . Testing should be carried out by suitably trained audiologists using modern calibrated technology ${ }^{31}$. Most young children less than 3.5 years with DS are unable to cooperate with conventional behavioural audiological testing ${ }^{25}$ Assessment with brain auditory evoked potentials (BAEP) is recommended in DS children ${ }^{25,32}$. It should be noted that the average latency values of BAEP established for healthy children should not be used as a reference for children with DS ${ }^{32}$ DS children have shortened latency values in BAEP which may be secondary to accelerated maturation of the nervous system or anatomical/functional disturbances of the central nervous system 32 The presence of narrow canals also predisposes to wax accumulation which may affect hearing and impedance testing ${ }^{33}$ Recommended monitoring protocol includes screening at birth followed by full assessment by 10 months of age to include auditory thresholds, impedance testing and otoscopy. At 18 months of age a further review is carried out with testing annually thereafter until 5 years of age and then 2 yearly for life ${ }^{34}$. More frequent assessments and intervention may be necessary should a deficit be found ${ }^{34}$

All DS patients should receive behavioral audiological testing every 6 months and annually after the age of three [19]. If tympanic membranes cannot be visualized, a referral to an Otolaryngologist should be made with the possible need for reassessment every 3 months if canals are significantly stenotic [10]. Treatment of hearing loss from chronic ear disease should involve close follow-up to ensure resolution of effusions, with a low threshold to place PE tubes given the risks for speech and language delay and use of hearing of aids in cases of sensorineural hearing loss. Audiologic evaluations should follow the American Academy of Pediatrics practice guidelines to monitor this high-risk population, and amplification should be considered as an appropriate intervention option if repeated audiology examinations reveal hearing loss. Multiple studies showed the evidence that conductive hearing loss is more prevalent in DS population as compared SNHL. However longitudinal studies on large populations are required.

\section{REFERENCES}

1. Collins VR, Muggli EE, Riley M, et al. Is Down syndrome a disappearing birth defect? J Pediatr 2008;152:20-4.

2. Roizen NJ, Patterson D. Down's syndrome. Lancet 2003;361:1281-9.

3. Schepis C, Barone C, Siragusa M, et al. An updated survey on skin conditions in Down syndrome. Dermatology 2002;20:234-8.

4. Weijerman ME, van Furth AM, Vonk Noordegraaf A, et al. Prevalence, neonatal characteristics and firstyear mortality of Down syndrome: a national study. J Pediatrics 2008;152:15-9.

5. Irving C, Basu A, Richmond S, et al. Twenty-year trends in prevalence and survival of Down syndrome. Eur J Hum Genet 2008;16:1336-40

6. Shin M, Besser LM, Kucik JE, et al. Prevalence of Down Syndrome among children and adolescents in 10 regions of the United States. Pediatrics 2009;124:1565-71.

7. Skotko BG, Capone GT, Kishnani S. Postnatal diagnosis of Down syndrome: synthesis of the evidence on how best to deliver the news. Pediatrics 2009;124:e751-8.

8. Yang Q, Rasmussen SA, Friedma JM. Mortality associated with Down's syndrome in the USA from 1983 to 
1997: a population-based study. Lancet 2002;359:1019-25.

9. Zwaan MC, Reinhardt DR, Hizler J, Vyas P. Acute leukemias in children with Down syndrome. Pediatr Clin North Am 2008;55:53-70.

10. Guidelines for basic essential medical surveillance. The Down's syndrome medical interest group UK and Ireland.

11. Kishnani PS, Crissman BG. Special issue: current perspectives on Down syndrome: selected medical and social issues. Am J Med Genet C Semin Med Genet 2006;142C:127-205.

12. D.M. Schwartz, R.H. Schwartz, Acoustic impedence and otoscopic findings in young children with downs syndrome, Arch. Otolaryngol. 104 (1978) 652-656.

13. M. Strome, Down's syndrome: a modern otorhinolaryngological perspective, Laryngoscope 41 (1981) 1581-1594.

14. S.R. Shott, Down syndrome: common otolaryngologic manifestations, Am. J. Med. Genet. Part C: Semin. Med. Genet. 142C (2006) 131-140

15. Marit Erna Austeng, Harriet Akre, Eva-Signe Falkenberg, Britt Øverland, Michael Abdelnoor, Kari Jorunn Kværner. Hearing level in children with Down syndrome at the age of eight. Research in Developmental Disabilities 34 (2013) 2251-2256

16. M.L. Casselbrant, L.M. Brostoff, E.I. Cantekin, M.R. Flaherty, W.J. Doyle, C.D. Bluestone, et al., Otitis media with effusion in preschool children, Laryngoscope 95 (1985) 428-436.

17. E. Barr, J. Dungworth, K. Hunter, M. McFarlane, H. Kubba, The prevalence of ear, nose and throat disorders in preschool children with Down's syndrome in Glasgow, Scott. Med. J. 56 (2011) 98-103.

18. S. Shott, A. Joseph, D. Heithaus, Hearing loss in children with Down syndrome, Int. J. Paediatr. Otorhinolaryngol. 61 (2001) 199-205.

19. Emily Nightengale, Patricia Yoon, Kristy Wolter-Warmerdam, Dee Daniels, and Fran Hickey. Understanding Hearing and Hearing Loss in Children with Down syndrome. American Journal of Audiology Vol. 26301-308 September 2017

20. A.H. Park, M.A. Wilson, P.T. Stevens, R. Harward, N. Hohler, Identification of hearing loss in pediatric patients with Down syndrome, Otolaryngol. Head Neck Surg. 146 (2012) 135-140

21. H.A. Kattan, R.F. Jarrar, Z.Z. Mahasin, A pilot study of the relationship between Down's syndrome and hearing loss, Saudi Med. J. 21 (2000) 931-933.

22. D.N. Brooks, H. Wooley, G.C. Kanjilal, Hearing loss and middle ear disorders patients with Down's syndrome (mongolism), J. Ment. Defic. Res. 16 (1972) 21-29.

23. S. Blaser, E.J. Propst, D. Martin, A. Feigenbaum, A.L. James, P. Shannon, et al., Inner ear dysplasia is common in children with Down syndrome (trisomy 21), Laryngoscope 116 (2006) 2113-2119.

24. L. De Schrijver, V. Topsakal, M. Wojciechowski, P. Van de Heyning, A. Boudewyns,Prevalence and etiology of sensorineural hearing loss in children with down syndrome: A cross-sectional study, International Journal of Pediatric Otorhinolaryngology, Volume 116,2019,Pages 168-172,

25. Nancy J. Roizen, Cheryl Wolters, Trent Nicol, and Thomas A. Blondis. Hearing loss in children with Down syndrome. The Journal of Pediatrics July 1993

26. Y. Iino, Y. Imamura, S. Harigai, Y. Tanaka, Efficacy of tympanostomy tube insertion for otitis media with effusion in children with Down syndrome, Int. J. Pediatr. Otorhinolaryngol. 49 (1999) 143-149.

27. L.M. Paulson, T.S. Weaver, C.J. Macarthur, Outcomes of tympanostomy tube placement in children with Down syndrome - a retrospective review, Int. J. Pediatr. Otorhinolaryngol. 78 (2014) 223-226

28. American Academy of Pediatrics, Committee on genetics. American Academy of Pediatrics: health supervision for children with Down syndrome, Pediatrics 107 (2001) 442-449.

29. Raut P, Sriram B, Yeoh A, Hee KY, Lim SB, Daniel ML. High prevalence of hearing loss in Down syndrome at first year of life. Ann Acad Med Singapore. 2011 Nov;40(11):493-8

30. Adelola OA, Papanikolaou V, Gormley P, Lang J, Keogh IJ, Newborn Hearing Screening: A Regional Example for National Care; IMJ, May 2010, vol 103, 5

31. Evenhuis,H.M. (1996) Dutch consensus on diagnosis and treatment of hearing impairment in children and adults with intellectual disability. J.Intel. Disabil. Res. 40(1) 451-456

32. Krecicki T, Zalesska-Krecicka M, Kubiak K, Gawron W. Brain auditory evoked potentials in children with Down syndrome. Int J Pediatr Otorhinolaryngol. 2005 May;69(5):615-20. Epub 2005 Jan 22.

33. Evenhuis,HM.,van Zanten,GA.,Brocnar,MP.,Roerdinkholder,WHM. Hearing loss in middleage persons with Down syndrome. Am.J.on Mental Retardation. 1992. 97.47-56.

34. Down's Syndrome Medical Interest Group Guidelines. Basic Medical Surveillance Essentials for People with Down's Syndrome, Hearing Impairment: 2007;9-11 